\title{
The Migrant Citizen-role Identity and the Relationship with Family Socioeconomic Status :the Mediating Role of Social Support
}

\author{
Shudan Feng ${ }^{1}$, Yang Liu $^{2,{ }^{*}}$, Qi Jiang ${ }^{3}$ \\ ${ }^{1 .}$ School of Humanlties and Management Sciences, Sichuan Medical University, Luzhou \\ 646000,China; \\ 2.Mental Health Center, the Affiliated Hospital of Southwest Medical University, Luzhou \\ 646000, China; \\ 3. Department of Psychology, Southwest University, Chongqing400715, China.
}

Key words: the migrant children; family socioeconomic status; citizen-role identity; social support; mediating role

\begin{abstract}
To explore the relationship between family socioeconomic status and citizen-role identity, and also the mediating role of social support, 547 students from six primary and middle schools in Chongqing city were selected to fill in questionnaires. The results indicated that: the migrant children and urban children did not exist significant differences in citizen-role identity; whether the migrant children or the urban children, social support plays partial mediating role between family socioeconomic status and citizen-role identity.
\end{abstract}

\section{Introduction}

Since the last decades in last century, a large amount of children migrant into and study in Urban from the countryside with their parents during the transformation of "single-model" or "couple-model" to "family-model" and being the migrant children in city. The Education department and the Police department co-promulgated "temporary rule for the migrant children study" in 1998, which defined the migrant children are those aged in 6-14 or 7-15 whose parents were lived temporarily in the migrant-in city for more than half year. The people's daily news reported the peasant-worker in the city are more than 200 million and followed 14 million children. (Guanghui Ni,2010).The scale of the migrant citizen is increasing with the further development of Chinese market economics and most of the migrant children would live in urban(State statistical bureau,2002). The cognition and emotional experience of the urban citizenship of those migrant children not only influence their urban adaption but also have a great infect on the persistent and further modernization. However, the previous studies were mainly focused on the identity which means the social position and the sense of group belonging in the migrant children(Ying Wang,2008), and the identification was considered as classification variable to explore the psychological and behavioral characteristics of the migrant children. Obviously, the definition of the urban people are changed with the further development of the Chinese urbanization which determined the study on the migrant children adaptation should not only focus on the social position and psychological and behavioral characteristics but a further study on the micro psychological development of the migrant citizen-roll identification, which means the construction of the citizen-roll and act as citizen consistency on mentally and behaviorally during the interaction of the migrant children and the urban life.(Wen Su,2011).Citizen-roll identification is an important mental 
function and a factor of new environment adaptation which can broaden one's social connection and play an positive roll in social interaction.

A variety of internal and external factors affect the role of citizen-role identity, among them, the major ones are socioeconomic (Jiliang Shen, 2007), teacher-student relationship(Juan R., Guardia Nancy J., \&Evans, 2008), peer relationship (Colette Sabatier, 2008), social support (Nerina L. Jimmieson, B. M. Mckimmie, Rachel L. Hannam, \& Jenifer Gallagher, 2008), and self-concept(Timothy B. Smith, Lynda Silva, 2011), and the socioeconomic of the family and the social support are attracted the most attention. The previous study indicated that the socioeconomic of the family have a great influence on the family member through the economic condition to restrict the development and effect of the mental health of the member.

Anthony D. Ong, Jean S. Phinney, \& Jessica Dennis (2006) pointed out SES is relevant to some dangerous factors such as hurting parents and community violence.Jiliang Shen(2007) stated that the socioeconomic state of the migrant children was generally low and poor living condition in city, so the urban living condition make the migrant children realized the huge difference between their birthplace and the city and the marginal adverse urban status of their parents to produce the roll identification crisis of the migrant children.

Social support also play a direct roll on the citizen-roll identification and influenced by the socioeconomic. Generally speaking, people can acquire resource from the social net dot from the social support and obtain care, respect and value from the social network member to reduce the individual life pressure to play a positive effect on the social adaptation and psychological health (Cobb, 1976). Social support has close relationship and has significant prediction of the citizen-roll identification (Nerina L. Jimmieson , B. M. Mckimmie, Rachel L. Hannam, \& Jenifer Gallagher, 2008). Due to the social support, the migrant children who has obtain social support can cope with discrimination ,the new unfamiliar culture ,identification of birthplace and urban are helped to find out their self value and erased their negative thought (Era Jng, Michael L. Echt, \& Brooke Chapman Wadsworth, 2008). Some study suggested that the level of the socioeconomic would influence the level of the social support(Jenny, Hsin- chun, \& Tsai. Xenophobi, 2006).The lower socioeconomic family restrict the individual social network development which lead to decrease of the social support from parents, school and peer, then has a negative effect on citizen-roll identification. The social support play a mediate roll in socioeconomic of the family and the citizen-roll identification , but there are few experimental evidence and most previous studies were mainly on the main effect and adjust effect, while less on the mediate function on social support(Wei Wang, Jing Luo,Wenbin Gao, 2010).

In a word, the socioeconomic of the family and social support have great influence on the citizen-roll identification of the migrant children, the socioeconomic work on the citizen-roll identification through the social support. Therefore, the present study mainly explore two questions: whether the social support play mediate roll between the socioeconomic and citizen-roll identification, and the comparison of the mediate function of the social support between migrant children and urban children.

\section{Method}

Subject. 547 primary and middle students from 6 schools in Chongqing, China were chose randomly .516(276 boys',240 girls', primary school students’308,middle school students'208 ) effective questionnaire were retrieved and the retrieved rate was 94.33\%.All the subject were divided into migrant children(235) and urban children(281) according to the answer "with the work parents to the city" or "to the city due to the transformation of the work parents" of the question 
"the reason to study in city", combined with the living time in the city had been more than half a year

Tools. The Socioeconomic status of family questionnaire: The Socioeconomic status of the family(SES) is measured from the parents career, educational degree and income. The calculation of the SES score were mainly in two ways in China, one is the simple plus way introduced by Baoguo Shuai and Jiliang Shen, the other way was introduced by Xiaojiao Yuan and Xiaoyi Fang(2009) according to 4 step calculation procedure of the 2003 program of international students assess (PISA).The questionnaire was modified from the Baoguo Shuai and Jiliang Shen's SES questionnaire and followed the same calculation method.

Social support scale:The social support scale (Xiangdong Wang,Xilin Wang,Hong Ma,1999)established by Shuiyuan Xiao was modified to apply in the present study. The structure of the scale include 3 dimension named subjective support, objective support, the usage of the support and 10 items. The sum of the score is bigger,which means they get more social support. The inner reliability of the social support scale is 0.92 in the present study.

The questionnaire of citizen-roll identification of migrant children:The current study employed self compiling questionnaire which include 20 items ranged from "totally disagree" to "totally agree” and count 1-5 score respectively, the higher score means more identification. The scale include 4 dimensions which includes information acquire, ability perception, positive emotion and behavior tendency and the $\alpha$ coefficient are $0.766,0.828,0.739$ and 0.652 of each dimension and the total $\alpha$ coefficient is 0.835 . The mainly goodness index of confirmatory factor analysis are $x$ 2=546.448, $\mathrm{df}=164, \mathrm{p}=0.000, \times 2 / \mathrm{df}=3.332, \mathrm{RMR}=0.076, \mathrm{CFI}=0.928, \mathrm{GFI}=0.953$.

Procedure. Questionnaire was employed in the current study. The Subjects are psychology graduate student under strict training to present the direction to group test. The objects were guided to read the direction carefully and finished the questionnaire according to the questionnaire requirements. No need to sign on the test and about 25 minutes are required to finish the test and all the questionnaire would be retrieval once they finished them.

Statistical tool. SPSS 17.0 and Amos 5.0

\section{Results and Analysis}

The comparison of the citizen-roll identification of migrant children and urban children. The independent T-test was employed to investment the difference between the citizen-roll identification of migrant children and urban children, the result indicated the mean of the citizen-roll identification of migrant children is 2.619,SD 0.545 , while the mean of the citizen-roll identification of the urban children is 2.548 ,SD $0.571, \mathrm{p}=.151>0.01$. which means there is no significant difference between the citizen-roll identification of migrant children and urban children.

The co-relationship analysis of socioeconomic status, citizen-roll identification and social support. Table 1 listed out all the variables and the correlation coefficient and the co-relationship between each variable reaches the statistical significance, to be specific, socioeconomic status positively co-relat with the citizen-roll identification which means higher the socioeconomic status, higher the citizen-roll identification. Social support also positively co-related with the citizen-roll identification. In addition, socioeconomic status, social support and the 3 dimensions have average or high positive relation. 
Table 1 The co-relation analysis among SES, citizen-roll identification, social support and corresponding dimensions

\begin{tabular}{cccccc}
\hline & $\begin{array}{c}\text { citizen-roll } \\
\text { identification }\end{array}$ & $\begin{array}{c}\text { social } \\
\text { support }\end{array}$ & $\begin{array}{l}\text { objective } \\
\text { support }\end{array}$ & $\begin{array}{l}\text { subjective } \\
\text { support }\end{array}$ & $\begin{array}{c}\text { the } \\
\text { support degrees }\end{array}$ \\
\hline social support & $0.30^{* *}$ & & & \\
objective support & $0.16^{*}$ & $0.76^{* *}$ & & \\
subjective support & $0.28^{* *}$ & $0.85^{* *}$ & $0.42^{* *}$ & & \\
the use of support & $0.27^{* *}$ & $0.75^{* *}$ & $0.39^{* *}$ & $0.47^{* *}$ & \\
degrees & & & & \\
SES & $0.21^{* *}$ & $0.27^{* *}$ & $0.27^{* *}$ & $0.23^{* *}$ & $0.13^{* *}$ \\
\hline
\end{tabular}

Note:**means $\mathrm{p}<0.01, *$ means $\mathrm{p}<0.05$ (the same below)

The test of the mediate function of the social support. The structure equal model was employed to analyze the relationship among the variables. According to the procedure of the mediate effect test (Zhonglin Wen,Lei Zhang,Jiehou Tai,2004; Preacher, Curran , \& Bauer, 2006),the direct effect of socioeconomic status to citizen-roll identification was tested first, then to test the goodness and the significance of the routine coefficient after the mediate variable added in.

The result indicated the routine coefficient of the direct effect of socioeconomic status to citizen-roll identification was significant $(\beta=0.21, \mathrm{SE}=0.08, \mathrm{p}<0.01)$. After the social support was added in the socioeconomic status and citizen-roll identification, the routine coefficient of the socioeconomic status and social support, socioeconomic status and citizen-roll identification, social support and citizen-roll identification were all significant, but the routine coefficient of the socioeconomic status and citizen-roll identification reduced from 0.21 to $0.11 \quad(t=0.12, S E=0.04$, $\mathrm{P}=0.004<0.05$ ）, which meeted the mediate effect require and presented indirect effect to influence the citizen-roll identification directly and indirectly effect through the social support. The chart 1 further indicated the indirect effect of the socioeconomic status to the citizen-roll identification reached $11.9 \%$, the proportion of the mediate effect to the total effect is $51.97 \%$.

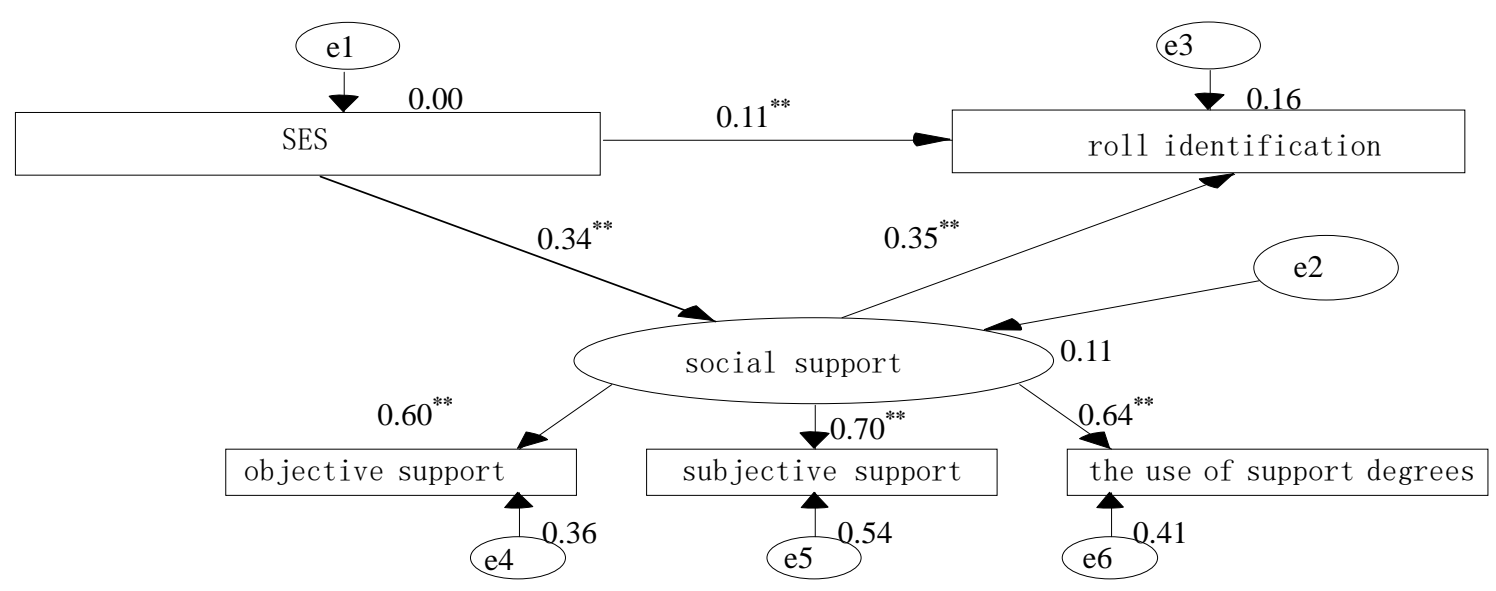

Fig. 1 The routine map of the partial mediate effect of social support on socioeconomic status and citizen-roll identification 
Table 2 The goodness of the structure model of the mediate effect of social support on socioeconomic status and citizen-roll identification

\begin{tabular}{ccccccccc}
\hline$\chi 2$ & $\mathrm{df}$ & $\chi 2 / \mathrm{df}$ & RMSEA & RMR & GFI & AGFI & TLI & PGFI \\
\hline 33.43 & 15 & 2.23 & 0.04 & 0.01 & 0.99 & 0.96 & 0.95 & 0.33 \\
\hline
\end{tabular}

\section{The comparison of the structure equal model between migrant children and urban children}

The multi structure equal model was employed to test the function of the social support on the socioeconomic status and citizen-roll identification and whether there was significant difference between the structure equal model of the migrant children and urban children. The following relevant models are defined:

Model one ( base model ) : To the various groups defined the same model structure.

Model two: To define the predict routine coefficient equal partial corresponding to the different group structure model based on model one.

Model three: To define all the routine coefficient equal to different group model based on the model two.

The result indicated that there was no significant difference on the mediate model of social support on socioeconomic status and citizen-roll identification between migrant children and urban children which means socioeconomic status influenced the citizen-roll identification through the social support directly and indirectly in all the children. But the value of the mediate effect of the social support was different in migrant children and urban children. The mediate effect of the social support was $10.14 \%$ in urban children, proportioned $50.37 \%$ in total effect, while the mediate effect was $10.24 \%$,proportioned $48.21 \%$ in total effect in the migrant children.

Table 3 The comparison of the structure equal model between migrant children and urban children

\begin{tabular}{ccccccc}
\hline$\Delta \chi$ & df & $p$ & NFI & IFI & RFI & TLI \\
\hline 4.58 & 3 & 0.21 & 0.01 & 0.01 & 0.00 & 0.00 \\
\hline
\end{tabular}

\section{Discussion}

The comparison of the citizen-roll identification between migrant children and urban children. The result of the independent $\mathrm{T}$ test indicated there was no significant difference in citizen-roll identification between migrant children and urban children which inconformity with the previous study(Jiliang Shen,Xinghua Wang,2008).The reason may lies in the specific sample, all the children were chosen in Chongqing,China which has a high confluence due to the historical specification. The outer culture is easily accepted and absorbed by the native citizen, so the migrant children may feel less discrimination and repulsion and obtained higher citizen-roll identification. The other reason is the migrant children entered urban with best wishes, they live in a lower socioeconomic status family compared with the urban children but a higher status than the hometown children. The citizen-roll identification is cognition oriented to guide them to overcome various difficulties.

The partial mediate effect of socioeconomic status and citizen-roll identification and social support. First of all, the present study confirmed the previous research from the relationship on the socioeconomic status and citizen-roll identification (Jiliang Shen,2007), the higher the socioeconomic status, the better citizen-roll identification. Then how did the socioeconomic status effect the citizen-roll identification? The study employed the social support as the mediate variable to explore the progress or mechanism of the socioeconomic status effect. The result indicated the 
social support played partial mediate effect in socioeconomic status and citizen-roll identification which means socioeconomic status can directly effect the citizen-roll identification or indirectly by the social support.

The previous research suggested that social support is consistent obtain or perception and consciously providing enough social resource may play positive function. It's a long interaction process for the migrant children to adapt the city. Though it is hard to change the socioeconomic status in a short time compared with the urban children but the social support plays as a mediate effect in socioeconomic status and roll identification and provides a routine for the migrant children to improve their roll identification. The following measures will play positive roll in the migrant children identification, such as the adjusted national policy, the improvement of the school condition, care from parents and teachers and acceptance of the peer.

Second, no matter the urban children or migrant children, the social support plays partial effect in socioeconomic status and citizen-roll identification which means there is no significant difference in the structure equal model of the two group. The level of the socioeconomic status has direct and indirect influence(two routines) on the citizen-roll identification and the indirect routine works from the social support to effect the citizen-roll identification. Though the current confirmed the social support is the mediate variable in socioeconomic status and citizen-roll identification, $\mathrm{T}$ test indicated there is significant difference on the objective support between migrant children and urban children, and the influence of the difference on urban adaptation of the migrant children need a further explore.

\section{Conclusion}

1. There is no significant difference on the citizen-roll identification of the migrant children and urban children.

2. The social support play partial mediate effect in socioeconomic status and citizen-roll identification both in migrant and urban children, but the level of the mediate effect was different.

\section{References}

[1] Anthony D. Ong, Jean S. Phinney, \& Jessica Dennis. (2006 ). Competence under Challenge: Exploring the Protective Influence of Parental Support and Ethnic identity in Latino college students. Journal of Adolescence, ( 29) , 961- 979.

[2]Baoguo Shuai,Jiliang Shen. ( 2007) . The relationship between Family socioeconomic status, intelligence, the internal motivation and creativity. The psychological development and education,23( 1), 30- 34.

[3]Colette Sabatier. ( 2008) . Ethnic and National Identity among Second- Generation Immigrant Adolescents in France: the Role of Social Context and Family.Journal of Adolescence, 31( 2) , 185205.

[4]Era Jng, Michael L. Echt, \& Brooke Chapman Wadsworth. ( 2007) . The Role of Identity in International Students' Psychological Well-being in the United States: A Model of Depression Level, Identity Gaps, Discrimination, and Acculturation. International Journal of Intercultural Relations, 31( 5) , 605- 624.

[5]Gilbert C. Geeetal. ( 2006) . Social Support as a Buffer for Perceived Unfair Treatment Among Filipino Americans:Difference Between San Francisco and Honolulu. American Journal of Public Health,(4). 
[6]Jenny, Hsin-chun, \& Tsai. ( 2006) . Xenophobi, Ethnic Community, and Immigrant Youths' Friendship Network Formation. Adolescence, 42( 162) , 285- 298.

[7]Jiliang Shen,Xinghua Wang. ( 2007) . what migrant means for children - thinking again to a psychology study. China Peter, (6), 27- 38.

[8]Guanghui Ni. ( 2010) . 14 million migrant children in China is expected to enter a higher school in city. The People's Daily, 3. 4.

[9]Juan R, Guardia Nancy J, \& Evans. ( 2008) . Factors Influencing the Ethnic Identity Development of Latino Fraternity Members at a Hispanic Serving Institution. Journal of College Student Development, 49( 3) .

[10]Nerinal. Jimmieson, B. M. Mckimmie, Rachel L. Hannam, \& Jenifer Gallagher. ( 2010) . An Investigation of the Stress- Buffering Effects of Social Support in the Occupational Stress Process as a Function of Team Identification. Group Dynamics: Theory, Research, and Practice, 14( 4), 350- 367.

[11]Population SheKeSi of the national bureau of statistics.( 2002) . The provinces of the migrant population is more than 40 million. China's statistical information network.

[12]Timothy B. Smith, Lynda Silva. ( 2011) . Ethnic Identity and Personal Well- being of People of Color: A Meta- Analysis. Journal of Counseling Psycology, 58( 1) , 42- 60.

[13]Wei Wang,Jing Luo, Yi Wang,Wenbin Gao. ( 2010) . The relationship between family factors, social support and emotional in migrant children. The behavioral medicine and brain science magazine, 19 (6) , 556- 557.

[14]Wen Su. ( 2011) .Study of the citizen-roll identification of the migrant children and its influencing factors . Master's thesis. Chongqing. Institute of education science in Southwest university.

[15]Xiaojiao Yuan,Xiaoyi Fang,Yang Liu,Zhiruo Li. ( 2009) . The relationship between education resettlement way and city adaptation in migrant children. Journal of Beijing Normal University, (5) , 25- 33.

[16]Xiangdong Wang,Xilin Wang,Hong Ma. ( 1999) . Mental health assessment handbook. Revised and enlarged edition. Beijing: Chinese Mental Health Journal.

[17]Ying Wang. Identity and identity construction research elucidated. Journal of Henan Normal University, 2008, 35(1), 50-54. 